\title{
Control and inverse problems for networks of vibrating strings with attached masses
}

\author{
F. Al-Musallam ${ }^{1}$, S. Avdonin ${ }^{2}$, N. Avdonina ${ }^{2}$, J. Edward ${ }^{3}$ \\ ${ }^{1}$ Department of Mathematics, Kuwait University, P.O Box 5969 Safat, Kuwait \\ ${ }^{2}$ Department of Mathematics and Statistics, University of Alaska, Fairbanks, AK 99775, USA \\ ${ }^{3}$ Department of Mathematics and Statistics, Florida International University, Miami, FL 33199, USA \\ musallam@sci.kuniv.edu.kw, s.avdonin@alaska.edu, navdonina@alaska.edu, edwardj@fiu.edu
}

\section{DOI 10.17586/2220-8054-2016-7-5-835-841}

We consider the control and inverse problems for serially connected and tree-like networks of strings with point masses loaded at the internal vertices. We prove boundary controllability of the systems and the identifiability of varying coefficients of the string equations along with the complete information on the graph, i.e. the loaded masses, the lengths of the edges and the topology (connectivity) of the graph. The results are achieved using the Titchmarch-Weyl function for the spectral problem and the Steklov-Poincaré operator for the dynamic wave equation on the tree. The general result is obtained by the leaf peeling method which reduces the inverse problem layer-by-layer from the leaves to the fixed root of the tree.

Keywords: wave equation on graphs, inverse problem, boundary control.

Received: 12 September 2016

\section{Introduction}

This paper concerns the control and inverse problems for differential equations on quantum graphs. By quantum graphs, we understand differential operators on geometric graphs coupled by certain vertex matching conditions. B.S. Pavlov and his former student N. I. Gerasimenko were among the first researchers to develop a mathematically rigorous approach to differential operators on metric graphs in the 1980's [1,2].

Network-like structures play a fundamental role in many scientific and engineering problems. The classical problem here that aries from applications is the problem of oscillations of the flexible structures made of strings, beams, cables, and struts. These models describe bridges, space-structures, antennas, transmission-line posts, steelgrid reinforcements and other typical objects of civil engineering. More recently, the applications on a much smaller scale have come into focus. In particular, hierarchical materials like ceramic or metallic foams, percolation networks and carbon and graphene nano-tubes, and graphene ribbons have attracted much attention.

Papers discussing differential and difference equations on graphs have been appearing in various areas of science and mathematics since the 1930's, but in the last two decades, their numbers have grown enormously. Quantum graphs arise as natural models of various phenomena in chemistry (free-electron theory of conjugated molecules), biology (genetic networks, dendritic trees), geophysics, environmental science, disease control, and even in the internet (internet or network tomography). In physics, interest in quantum graphs arose, in particular, from applications to nano-electronics and quantum waveguides. On the other hand, quantum graph theory gives rise to numerous challenging problems related to many areas of mathematics from combinatorics to PDEs and spectral theory. Work on quantum graph theory and its applications have truly interdisciplinary character, and a series of meetings on this topic has stimulated collaboration of researchers from different areas of science, engineering and mathematics. A number of surveys and collections of papers on quantum graphs have appeared recently, and the first book on this topic by Berkolaiko and Kuchment [3] contains an excellent list of references.

Control and inverse theories constitute important parts of this rapidly developing area of applied mathematics - analysis on graphs. It is tremendously important for all aforementioned applications. However, these theories have not been sufficiently developed. Control and inverse problems for DEs on graphs appear to be much more complicated than similar problems on an interval (see, e.g. [4,5] and references therein).

A new effective leaf-peeling method for solving inverse problems for differential equations on graphs without cycles has been proposed in [5] and developed further in [6,7]. The main goal of the present paper is to extend this method to DEs on graphs with attached point masses.

Let $\Gamma=E \cup V$ be a finite compact metric graph without cycles, where $E=\left\{e_{j}\right\}_{j=1}^{N}$ is a set of edges and $V=\left\{\nu_{j}\right\}_{j=1}^{N+1}$ is a set of vertices. We recall that a graph is called a metric graph if every edge $e_{j} \in E$ is identified with an interval $\left(a_{2 j-1}, a_{2 j}\right)$ of the real line with a positive length $l_{j}=\left|a_{2 j-1}-a_{2 j}\right|$, and a graph is a tree if it 
has no cycles. The edges are connected at the vertices $v_{j}$ which can be considered as equivalence classes of the edge end points $\left\{a_{j}\right\}$.

Let $\left\{\gamma_{1}, \ldots, \gamma_{m}\right\}=\partial \Gamma \subset V$ be the boundary vertices, i.e. if the index (or multiplicity) of a vertex, $i d(\nu)$, is the number of edges incident to it, then $\partial \Gamma=\{\nu \in V \mid i d(\nu)=1\}$. A nonnegative mass $M_{\nu}$ is attached to each vertex $\nu \in V \backslash \partial \Gamma$.

In Fig. 1 we give an example of a star graph (a graph with one internal vertex). Such graphs play an important role in the leaf peeling method described below in Sec. 3. A tree with $m=9$ and $N=12$ is presented in Fig. 2.

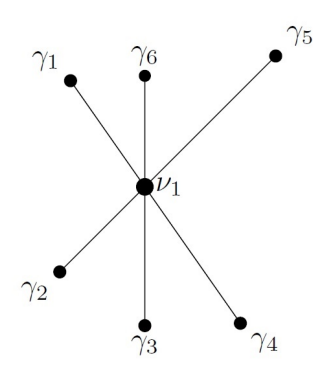

FIG. 1. A star graph

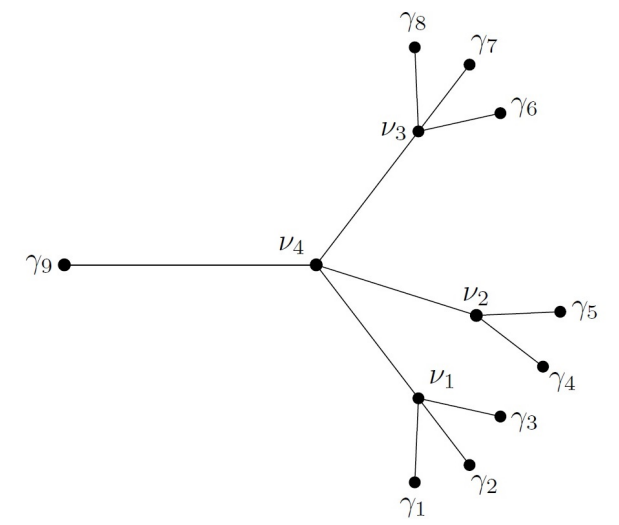

FIG. 2. A metric tree

Let $q$ be a continuous function on $\Gamma$. Our initial boundary value problem is:

$$
\begin{gathered}
u_{t t}-u_{x x}+q(x) u=0 \text { in }\{\Gamma \backslash V\} \times(0, T) \\
\sum_{e_{j} \sim \nu} \partial u_{j}(\nu, t)=M_{\nu} u_{t t}(\nu, t) \text { at each vertex } \nu \in V \backslash \partial \Gamma, \text { and } t \in[0, T] \\
u(\cdot, t) \text { is continuous at each vertex, for } t \in[0, T] \\
u=f \text { on } \partial \Gamma \times[0, T] \\
\left.u\right|_{t=0}=0 \text { in } \Gamma .
\end{gathered}
$$

In (1.2) (and below), $\partial u_{j}(\nu)$ denotes the derivative of $u$ at the vertex $\nu$ taken along the edge $e_{j}$ in the direction outwards from the vertex. Also, $e_{j} \sim \nu$ means edge $e_{j}$ is incident to the vertex $\nu$, and the sum is taken over all edges incident to $\nu$. Since $\partial \Gamma$ consists of $m$ vertices, $f$ can be naturally identified with a function acting from $[0, T]$ to $\mathbb{R}^{m}$.

The metric graph $\Gamma$ determines naturally the Hilbert space of square integrable functions $\mathcal{H}=L^{2}(\Gamma)$. We define the space $\mathcal{H}^{1}$ of continuous functions $v$ on $\Gamma$ such that $v_{l_{e}} \in H^{1}(e)$ for every $e \in E$.

The $f$ appearing in (1.3) is the (boundary) control for the problem (1.1)-(1.4), and a solution to (1.1)-(1.4) will be denoted $u^{f}$. One can prove that for $f \in \mathcal{F}^{T}:=L^{2}\left([0, T] ; \mathbb{R}^{m}\right)$, the generalized solution $v^{f}$ of (1.1)-(1.4) belongs to $C([0, T] ; \mathcal{H})$ (see Theorem 1 below), and the control operator $W^{T}: \mathcal{F}^{T} \rightarrow \mathcal{H}$, given by $W^{T} f:=u^{f}(\cdot, T)$ is bounded. by:

The response operator (Steklov-Poincaré operator) for the system, $R^{T}=\left\{R_{i j}^{T}\right\}_{i, j=1}^{m}$, defined on $\mathcal{F}^{T}$ is defined

$$
\left(R^{T} f\right)(t)=\left.\partial u^{f}(\cdot, t)\right|_{\partial \Gamma}, \quad 0<t<T .
$$

Our dynamic inverse problem is to recover the unknown coefficient $q(x)$ on each edge of the graph from the response operator $R^{T}$. We can also recover the graph topology, all $M_{\nu}, \nu \in V \backslash \partial \Gamma$, and the lengths of all the edges. We can actually do this with the reduced operator $\left\{R_{i j}^{T}\right\}_{i, j=1}^{m-1}$. That is, the method has the flexibility of not needing the control and observation at one of the boundary vertices. We prove the dynamic inverse problem has a unique solution for sufficiently large $T$ (see Theorem 2 below) and give a constructive method for finding it.

Applying formally the Fourier-Laplace transform

$$
g \mapsto \int_{0}^{\infty} g(t) e^{i \omega t} d t
$$


to equations (1.1)-(1.3), we obtain the following boundary value problem depending on a complex parameter $\lambda=\omega^{2}$ :

$$
\begin{gathered}
-\phi_{x x}(x, \lambda)+q(x) \phi(x, \lambda)=\lambda \phi(x, \lambda) \text { on }\{\Gamma \backslash V\}, \\
\sum_{e_{j} \sim \nu} \partial \phi_{j}(\nu, \lambda)=-\lambda M_{\nu} \phi(\nu, \lambda) \text { at each vertex } \nu \in V \backslash \partial \Gamma, \\
\phi(\cdot, \lambda) \text { is continuous at each vertex, }
\end{gathered}
$$

The system of differential equations (1.6), (1.7) with zero Dirichlet boundary condition has only a trivial solution for $\lambda \notin \mathbb{R}$. Therefore, for any $\alpha \in \mathbb{C}^{m}$, this system of equations has a unique solution, $\phi^{\alpha}(x, \lambda)$, satisfying non-zero boundary conditions:

$$
\phi^{\alpha}\left(\gamma_{j}, \lambda\right)=\alpha_{j}, \quad j=1,2, \ldots, m, \quad \alpha=\operatorname{col}\left\{\alpha_{1}, \ldots, \alpha_{m}\right\}
$$

The $m \times m$ matrix $\mathcal{M}(\lambda)$ defined by $\mathcal{M}(\lambda) \alpha=\left.\partial \phi^{\alpha}\right|_{\partial \Gamma}$ is called the Titchmarsh-Weyl matrix function, or the TW-function. The TW-function is also known as the (spectral) Dirichlet-to-Neumann map. The TW-function $\mathcal{M}(\lambda)$ known for $\Im \lambda>0$ will play the role of the spectral data for solving boundary inverse problems on graphs.

\section{Main results}

In the case of a string with loaded masses it was noticed $[8,9]$ that the wave transmitted through a mass is more regular than the incoming wave. A similar effect also occurs for networks of strings. To formulate the result, we need the following definition. Among all paths from edge $e_{i}$ to the boundary vertex $\gamma_{j}$, let the degree, $d_{i}$, of the edge $e_{i}$ be the minimal (with respect to $j$ ) number of nonzero loaded masses on the path. For the following theorem we assume that $\left.q\right|_{\overline{e_{j}}} \in C^{d_{j}}\left(\overline{e_{j}}\right)$.

Theorem 1. Assume $M_{\nu}>0$ for all $\nu$. If $f \in \mathcal{F}^{T}$, then for any $t \in[0, T], u^{f}(\cdot, t) \in \mathcal{H}$ and $u^{f} \in C([0, T] ; \mathcal{H})$. Furthermore, for each $e_{j} \in E,\left.u^{f}\right|_{e_{j}} \in C\left([0, T] ; H^{d_{j}}\left(e_{j}\right)\right)$.

The proof of the theorem is based on the analysis of the waves incoming to, transmitted through and reflected from an inner vertex, taking into account the conditions (1.2). For the simplest graph of serially connected strings with attached masses such a result was obtained in [8].

The next theorem describes the solution of the dynamic inverse problem.

Theorem 2. Let $T_{*}=2 \max _{j \neq m}$ dist $\left\{\gamma_{j}, \gamma_{m}\right\}$. The operator $\left\{R_{i j}^{T}\right\}_{i, j=1}^{m-1}$ known for $T>T_{*}$ uniquely determines $q$ on $\Gamma,\left\{M_{\nu}: \nu \in V \backslash \partial \Gamma\right\},\left\{l_{j}: j=1, \ldots, N\right\}$ and the graph topology. If the topology is known, all other parameters can be found from the main diagonal $\left\{R_{i i}^{T}\right\}_{i=1}^{m-1}$ of the reduced response operator.

We also extend to our networks the leaf peeling method proposed in [5] (and generalized for strings with attached masses in [10]) and develop a constructive algorithm solving the inverse problem.

A spectral analog of Theorem 2 reads as follows.

Theorem 3. The reduced $T W$ matrix function $\left\{\mathcal{M}_{i j}(\lambda)\right\}_{i, j=1}^{m-1}$ known for $\Im \lambda>0$ uniquely determines $q$ on $\Gamma$, $\left\{M_{\nu}: \nu \in V \backslash \partial \Gamma\right\},\left\{l_{j}: j=1, \ldots, N\right\}$ and the graph topology. If the topology is known, all other parameters can be found from the main diagonal $\left\{\mathcal{M}_{i i}(\lambda)\right\}_{i=1}^{m-1}$ of the reduced TW matrix function.

\section{Proof of Theorem 3}

The response operator $R^{T}$ and TW-function $\mathcal{M}(\lambda)$ are connected with each other by the Fourier-Laplace transform (see, e.g. [5]). Therefore, knowledge of $\mathcal{M}(\lambda)$ allows one to find $R^{T}$ for all $T>0$, and knowledge of $R^{T}$ for all $T>0$ allows one to find $\mathcal{M}(\lambda)$.

In this section, we prove Theorem 3. We will give a brief description of an algorithm which allows us to recalculate the TW matrix function from the original graph to a smaller graph by "pruning" boundary edges. Ultimately, doing so allows us to reduce the original inverse problem on the graph to the inverse problem on a single interval.

Our reduction algorithm combines both spectral and dynamical approaches, i.e. uses $\mathcal{M}(\lambda)$ and $R^{T}$. As we mentioned above, the TW matrix function determines the response operator for the system (1.1)-(1.4). Therefore, under the conditions of Theorem 3 the entries $R_{i j}^{T}, i, j=1, \ldots, m-1$ are known for $T>0$.

Step 1. Knowledge of $R_{j j}^{T}$ for sufficiently large $T$ allows one to recover the length of the edge $e \in E$ incident to $\gamma_{j}$, the potential $q$ on $e$ and the mass $M_{\nu}$, where $\nu \in V \backslash \partial \Gamma$ is an inner vertex to which $e$ is incident. We can also recover $i d(\nu)$, the total number of edges incident to $\nu$. The proof of these statements is based on the analysis 
of the waves incoming to, transmitted through and reflected from vertex $\nu$. Similar analysis was presented in [5] without the loaded masses; this was based on the boundary control method in inverse theory.

Step 2. We determine the boundary edges which have a common end point using the non-diagonal entries $R_{i j}^{T}$ of the response operator. Since the speed of wave propagation in the system (1.1)-(1.4) equals one, two boundary edges, say, $e_{i}$ and $e_{j}$, incident to the boundary edges $\gamma_{i}$ and $\gamma_{j}$ with the lengths $l_{i}$ and $l_{j}$ have a common end point if and only if:

$$
R_{i j}^{T}= \begin{cases}0 & \text { for } T<l_{i}+l_{j} \\ \neq 0, & \text { for } T>l_{i}+l_{j} .\end{cases}
$$

Definition of a sheaf. We consider a subgraph of $\Gamma$ which is a star graph consisting of all edges incident to an internal vertex $v$. This star graph is called a sheaf if all but one its edges are the boundary edges of $\Gamma$. It is known that any tree has at least two sheaves.

Step 3. Leaf peeling. We consider now a sheaf consisting, say, of several boundary edges $e_{1}, \ldots, e_{p}, p<m$, incident to boundary vertices $\gamma_{1}, \ldots, \gamma_{p}$ are connected at the vertex $\nu_{s}$ (see, e.g. vertices $\gamma_{1}, \gamma_{2}, \gamma_{3}, \nu_{1}$ on Fig. 2). From Step 1, we know the potential on these edges, their lengths and the index of the vertex $\nu_{s}$.

The index of the vertex $\nu_{s}$ is $p+1$ and there is exactly one internal edge incident to $\nu_{s}$. We denote by $\widetilde{\mathcal{M}}(\lambda)$ the TW matrix function associated with the reduced graph $\widetilde{\Gamma}$, i.e. the original graph $\Gamma$ without the boundary edges $e_{1}, \ldots, e_{p}$ and vertices $\gamma_{1}, \ldots, \gamma_{p}$.

We denote by $\widetilde{\mathcal{M}}_{0 i}(\lambda), \widetilde{\mathcal{M}}_{i 0}(\lambda)$ and $\widetilde{\mathcal{M}}_{00}(\lambda)$ the entries of $\widetilde{\mathcal{M}}(\lambda)$ related to the "new" boundary point $\nu_{s_{0}}$ of the graph $\widetilde{\Gamma}$. The other entries of $\widetilde{\mathcal{M}}(\lambda)$ are denoted by $\widetilde{\mathcal{M}}_{i j}, i, j=p+1, \ldots, m$. We demonstrate now how to find the entries of $\widetilde{\mathcal{M}}(\lambda)$.

First, we recalculate the entries $\widetilde{\mathcal{M}}_{00}(\lambda)$ and $\widetilde{\mathcal{M}}_{0 i}(\lambda), i=p+1, \ldots, m-1$. we choose a boundary point, say $\gamma_{1}$, of the star-subgraph. Let $\phi(x, \lambda)$ be the solution to the problem (1.6), (1.7) subject to the boundary conditions:

$$
\phi\left(\gamma_{1}, \lambda\right)=1, \quad \phi\left(\gamma_{j}, \lambda\right)=0, \quad j=2, \ldots, m-1, m .
$$

We notice that on the boundary edge $e_{1}$ the function $\phi$ solves the Cauchy problem:

$$
\begin{array}{r}
-\phi^{\prime \prime}+q(x) \phi=\lambda \phi, \quad x \in e_{1}, \\
\phi\left(\gamma_{1}, \lambda\right)=1, \phi^{\prime}\left(\gamma_{1}, \lambda\right)=\mathcal{M}_{11}(\lambda) .
\end{array}
$$

On the other edges of the star subgraph it solves

$$
\begin{array}{r}
-\phi^{\prime \prime}+q(x) \phi=\lambda \phi, \quad x \in e_{i}, \quad i=2, \ldots, p, \\
\phi\left(\gamma_{i}, \lambda\right)=0, \phi^{\prime}\left(\gamma_{i}, \lambda\right)=\mathcal{M}_{1 i}(\lambda), \quad i=2, \ldots, p .
\end{array}
$$

Since the potential on the edges $e_{1}, \ldots, e_{p}$ is known, we can solve the Cauchy problems (3.3), (3.4) and (3.5), (3.6) and use the matching conditions (1.7) at the internal vertex $\nu_{s}$ to recover the values $\phi\left(\nu_{s}, \lambda\right)$ and $\phi^{\prime}\left(\nu_{s}, \lambda\right)$ on the "new" boundary edge at the "new" boundary point $\nu_{s}$. Thus we obtain:

$$
\begin{aligned}
& \widetilde{\mathcal{M}}_{00}(\lambda)=\frac{\phi^{\prime}\left(\nu_{s}, \lambda\right)}{\phi\left(\nu_{s}, \lambda\right)}, \\
& \widetilde{\mathcal{M}}_{0 i}(\lambda)=\frac{\mathcal{M}_{1 i}(\lambda)}{\phi\left(\nu_{s}, \lambda\right)}, \quad i=p+1, \ldots, m .
\end{aligned}
$$

We recall that here $\Im \lambda \neq 0$, and so, $\phi\left(\nu_{s}, \lambda\right) \neq 0$. Otherwise, $\lambda$ would be an eigenvalue of a selfadjoint operator.

To find $\widetilde{\mathcal{M}}_{i 0}(\lambda)$ and $\widetilde{\mathcal{M}}_{i j}(\lambda), i=p+1, \ldots, m-1$ we fix $\gamma_{i}(i>p)$ and consider the solution $\psi(x, \lambda)$ to (1.6), (1.7) with boundary conditions

$$
\psi\left(\gamma_{i}, \lambda\right)=1, \quad \psi\left(\gamma_{j}, \lambda\right)=0, \quad j \neq i .
$$

The function $\psi$ then solves the following Cauchy problems on the edges $e_{1}, \ldots, e_{p}$ :

$$
\begin{array}{r}
-\psi^{\prime \prime}+q(x) \psi=\lambda \psi, \quad x \in e_{j}, \quad j=1, \ldots, p, \\
\psi\left(\gamma_{j}, \lambda\right)=0, \psi^{\prime}\left(\gamma_{j}, \lambda\right)=\mathcal{M}_{i j}(\lambda) .
\end{array}
$$

Since we know the potential on the edges $e_{1}, \ldots, e_{p}$, we can solve the Cauchy problems (3.9), (3.10) and use the conditions at the internal vertex $\nu_{s}$ to recover the values $\psi\left(\nu_{s}, \lambda\right)$ and $\psi^{\prime}\left(\nu_{s}, \lambda\right)$ at the "new" boundary edge with the "new" boundary point $\nu_{s}$.

Now, we consider the following linear combination of the solutions $\phi$ and $\psi$ :

$$
\varphi(x, \lambda)=\psi(x, \lambda)-\frac{\psi\left(\nu_{s}, \lambda\right)}{\phi\left(\nu_{s}, \lambda\right)} \phi(x, \lambda) .
$$


It is easy to verify that on the subgraph $\widetilde{\Gamma}$ the function $\varphi$ satisfies the boundary conditions:

$$
\varphi\left(\gamma_{i}, \lambda\right)=1, \varphi\left(\gamma_{j}, \lambda\right)=0, j \neq i \text {. }
$$

Thus, from (3.11), we obtain that:

$$
\begin{aligned}
& \widetilde{\mathcal{M}}_{i 0}(\lambda)=\psi^{\prime}\left(\nu_{s}, \lambda\right)-\psi\left(\nu_{s}, \lambda\right) \widetilde{\mathcal{M}}_{00}(\lambda), \\
& \widetilde{\mathcal{M}}_{i j}(\lambda)=\mathcal{M}_{i j}(\lambda)-\psi\left(\nu_{s}, \lambda\right) \widetilde{\mathcal{M}}_{0 j}(\lambda) .
\end{aligned}
$$

To recover all elements of the reduced TW matrix function, we need to use this procedure for all $i, j=p+$ $1, \ldots, m-1$.

We conclude that the (reduced) TW-function for the graph $\Gamma$ determines the (reduced) $T W$-function for the graph $\widetilde{\Gamma}$. The inverse problem is reduced to the inverse problem for a smaller graph. Since the graph $\widetilde{\Gamma}$ is finite, this procedure may be continued, but it ends after a finite number of steps.

The proofs of Theorems 1 and 2 for arbitrary tree will be presented in a forthcoming paper. The quantum graph with the simplest topology - a network of serially connected strings - is considered in the next section.

\section{Network of serially connected strings}

We consider the wave equation on the interval [0, $\ell]$ with $N$ masses $M_{j}>0$ attached at the points $a_{j}, j=$ $1, \ldots, N$, where $0=a_{0}<a_{1}<\ldots<a_{N}<a_{N+1}=\ell$. This is modeled by:

$$
\begin{gathered}
\rho(x) \frac{\partial^{2} u}{\partial t^{2}}-\frac{\partial^{2} u}{\partial x^{2}}=0, t \in(0, T), x \in \Omega:=\left(0, a_{1}\right) \cup\left(a_{1}, a_{2}\right) \cup \ldots \cup\left(a_{N}, \ell\right), \\
u\left(a_{j}^{-}, t\right)=u\left(a_{j}^{+}, t\right), \quad M_{j} u_{t t}\left(a_{j}, t\right)=u_{x}\left(a_{j}^{+}, t\right)-u_{x}\left(a_{j}^{-}, t\right), \\
u(x, 0)=u_{t}(x, 0)=0, \\
u(0, t)=f(t), f \in \mathcal{F}^{T}:=L^{2}(0, T), \quad u(\ell, t)=0 .
\end{gathered}
$$

Here $\rho$ is a positive function on $[0, \ell]$ and $\left.\rho\right|_{\left[a_{j}, a_{j+1}\right]} \in C^{j+2}\left[a_{j}, a_{j+1}\right], j=0, \ldots, N$.

We show that the wave transmitted through a mass is more regular than the incoming wave. We define the spaces $W, W^{T}$ :

$$
\begin{gathered}
W=\left\{\phi \in L^{2}\left(0, a_{1}\right) \times H^{1}\left(a_{1}, a_{2}\right) \times \ldots H^{N}\left(a_{N}, \ell\right):\right. \\
\left.\phi\left(a_{j}^{-}\right)=\phi\left(a_{j}^{+}\right), \phi^{\prime}\left(a_{j}^{-}\right)=\phi^{\prime}\left(a_{j}^{+}\right)-M_{j} \phi^{\prime \prime}\left(a_{j}^{+}\right) / \rho\left(a_{j}^{+}\right), \phi(\ell)=0\right\}, \\
W^{T}=\{\phi \in W: \phi(x)=0 \text { for } x \geq X(T)\},
\end{gathered}
$$

where

$$
T=\int_{0}^{X(T)} \sqrt{\rho(x)} d x, \quad L=\int_{0}^{l} \sqrt{\rho(x)} d x .
$$

The following result on the regularity of the solution of the initial boundary value problem stated above and on the controllability of this dynamical system has been proved in [8].

Theorem 4. Suppose $T \leq L:=\int_{0}^{l} \sqrt{\rho(x)} d x$. For any $f \in \mathcal{F}^{T}, u^{f} \in C\left(0, T ; W^{T}\right)$ and for any $\phi \in W^{T}$, there exists a unique $f \in W^{T}$ such that $u^{f}(x, T)=\phi(x)$. Furthermore,

$$
\left\|u^{f}(\cdot, T)\right\|_{W} \asymp\|f\|_{\mathcal{F}^{T}} .
$$

For $T>L$

$$
\left\{u^{f}(\cdot, T): f \in L^{2}(0, T)\right\}=W .
$$

Our dynamical inverse problem is to recover unknown parameters of the system from the response operator

$$
\begin{gathered}
R^{T}: \mathcal{F}^{T} \mapsto \mathcal{F}^{T}, \operatorname{Dom}\left(R^{T}\right)=\left\{f \in H^{1}(0, T), f(0)=0\right\}, \\
\left(R^{T} f\right)(t)=u_{x}^{f}(0, t), t \in(0, T) .
\end{gathered}
$$

The main result is this section is:

Theorem 5. Let $T>2 L$. Given $R^{T}$, one can find $\rho(x), l, a_{j}$, and $M_{j}, j=1, \ldots, N$. 
We prove this theorem in several steps. First, we consider the spectral boundary value problem corresponding our dynamical system:

$$
\begin{gathered}
-\varphi^{\prime \prime}(x, \lambda)=\lambda^{2} \rho(x) \varphi(x, \lambda), \quad x \in \Omega, \\
\varphi(0, \lambda)=\varphi(\ell, \lambda)=0, \quad \varphi\left(a_{j}^{-}, \lambda\right)=\varphi\left(a_{j}^{+}, \lambda\right), \\
-M_{j} \lambda^{2} \varphi\left(a_{j}, \lambda\right)=\varphi^{\prime}\left(a_{j}^{+}, \lambda\right)-\varphi^{\prime}\left(a_{j}^{-}, \lambda\right) \quad \forall j .
\end{gathered}
$$

The eigenvalues $\lambda_{n}^{2}$ of this problem are simple and the eigenfunctions $\varphi_{n}$ form the orthonormal basis in the space $\mathcal{H}:=L_{\rho}^{2}(0, l) \oplus \mathcal{R}^{N}$ with the inner product:

$$
\langle\phi, \psi\rangle_{\mathcal{H}}=\int_{0}^{l} \phi(x) \psi(x) \rho(x) d x+\sum_{j=1}^{N} M_{j} \phi\left(a_{j}\right) \psi\left(a_{j}\right) .
$$

We set

The connecting operator is defined as:

$$
\mathcal{H}^{T}=\{\phi \in \mathcal{H}: \phi(x)=0 \text { for } x \geq X(T)\} .
$$

$$
C^{T}: \mathcal{F}^{T} \mapsto \mathcal{F}^{T}, \quad\left(C^{T} f, g\right)_{\mathcal{F}^{T}}:=\left\langle u^{f}(\cdot, T), u^{g}(\cdot, T)\right\rangle_{\mathcal{H}} .
$$

The connecting operator can be written in the form $C^{T}=\left(U^{T}\right)^{*}\left(U^{T}\right)$ where

$$
U^{T}: \mathcal{F}^{T} \mapsto W^{T}, U^{T} f=u^{f}(\cdot, T) .
$$

The exact controllability (see Theorem 4 ) implies that $C^{T}$ is bounded and boundedly invertible.

Our second step is:

Theorem 6. Operator $C^{T}$ can be explicitly expressed through the response operator on the double interval: $C^{T}=-\frac{1}{2}\left(S^{T}\right)^{*} I^{2 T} R^{2 T} S^{T}$, where:

$$
\left(S^{T} f\right)(t)=\left\{\begin{array}{ll}
f(t) & \text { if } t \in[0, T], \\
-f(2 T-t) & \text { if } t \in(T, 2 T],
\end{array} \quad\left(I^{2 T} f\right)(t)=\int_{0}^{t} f(s) d s\right.
$$

Sketch of the proof of Theorem 6. Set $w(s, t):=\left\langle u^{f}(\cdot, s), u^{g}(\cdot, t)\right\rangle_{\mathcal{H}}$.

We notice that $\left(C^{T} f, g\right)_{\mathcal{F} T}=w(T, T$ and

$$
\begin{aligned}
& w_{t t}(s, t)-w_{s s}(s, t)=\int_{0}^{l}\left[u^{f}(x, s) u_{t t}^{g}(x, t)-u_{s s}^{f}(x, s) u^{g}(x, t)\right] \rho(x) d x \\
+ & \sum_{j} M_{j}\left[u^{f}\left(a_{j}, s\right) u_{t t}^{g}\left(a_{j}, t\right)-u_{s s}^{f}\left(a_{j}, s\right) u^{g}\left(a_{j}, t\right)\right]=\left(\operatorname{using} \rho u_{t t}=u_{x x}\right) \\
= & {\left[u^{f}(x, s) u_{x}^{g}(x, t)-u_{x}^{f}(x, s) u^{g}(x, t)\right]_{x=0}^{l}=(R f)(s) g(t)-f(s)(R g)(t) . }
\end{aligned}
$$

We use $w(s, 0)=w_{t}(s, 0)=w(0, t)=0$ to find $w(T, T)$ by D'Alembert's formula.

The next step is the construction of special bases in spaces $\mathcal{F}^{T}$ and $\mathcal{H}^{T}$. Let $T \leq L$ and $\left\{f_{n}\right\}, n \in \mathbb{N}$, be a basis in $\mathcal{F}^{T}$ such that:

$$
f \in C^{2}[0, T], f(0)=f^{\prime}(0)=0, \quad\left(C^{T} f_{k}, f_{n}\right)_{\mathcal{F}^{T}}=\delta_{k n} .
$$

Due to controllability, $\left\{u^{f_{n}}(\cdot, T)\right\}$ is an orthonormal basis in $\mathcal{H}^{T}$.

Next, we introduce two functions: $\phi^{0}(x)=1, \phi^{1}(x)=x, x \in[0, l]$ and let $\phi_{T}^{0}$ and $\phi_{T}^{1}$ be their restrictions to the interval $[0, X(t)]$.

Theorem 7. The coefficients in the series representations of the functions $\phi_{T}^{0}, \phi_{T}^{1}$ with respect to the basis $\left\{u^{f_{j}}(\cdot, T)\right\}$ have the form

$$
\begin{gathered}
c_{n}^{0}:=\left\langle\phi^{0}, u^{f_{n}}(\cdot, T)\right\rangle_{\mathcal{H}}=-\int_{0}^{T}(T-t)\left(R^{T} f_{n}\right)(t) d t \\
c_{n}^{1}:=\left\langle\phi^{1}, u^{f_{n}}(\cdot, T)\right\rangle_{\mathcal{H}}=\int_{0}^{T}(T-t) f_{n}(t) d t .
\end{gathered}
$$


Sketch of the proof of Theorem 7. By the definition of the connection operator, we have:

$$
\begin{aligned}
& \left\langle\phi^{0}, u^{f_{n}}(\cdot, T)\right\rangle_{\mathcal{H}}=\int_{0}^{l} u^{f_{n}}(x, T) \rho(x) d x+\sum_{j} M_{j} u^{f_{n}}\left(a_{j}, T\right) \\
& =\int_{0}^{T}(T-t)\left[\int_{0}^{X(T)} u_{t t}^{f_{n}}(x, t) \rho(x) d x+\sum_{j} M_{j} u_{t t}^{f_{n}}\left(a_{j}, t\right)\right] d t \\
& =\int_{0}^{T}(T-t)\left[\int_{0}^{X(T)} u_{x x}^{f_{n}}(x, t) d x+\sum_{j} M_{j} u_{t t}^{f_{n}}\left(a_{j}, t\right)\right] d t \\
& =-\int_{0}^{T}(T-t) u_{x}^{f_{n}}(0, t) d t=-\int_{0}^{T}(T-t)\left(R^{T} f_{n}\right)(t) d t .
\end{aligned}
$$

This proves the first statement of Theorem 7. The second one can be proved in a similar way.

Now, we are ready to complete the solution of the dynamical inverse problem. We introduce two functions:

$$
\begin{gathered}
\mu(T)=\int_{0}^{X(T)} \rho(x) d x+\sum_{j: a_{j}<X(T)} M_{j}, \\
\nu(T)=\int_{0}^{X(T)} x \rho(x) d x+\sum_{j: a_{j}<X(T)} M_{j} a_{j} .
\end{gathered}
$$

They can be found using the theorem:

$$
\mu(T)=\left\langle\phi_{T}^{0}, \phi_{T}^{0}\right\rangle_{\mathcal{H}}=\sum_{n}\left|c_{n}^{0}\right|^{2}, \quad \nu(T)=\left\langle\phi_{T}^{0}, \phi_{T}^{1}\right\rangle_{\mathcal{H}}=\sum_{n} c_{n}^{0} c_{n}^{1} .
$$

Separating the singular and regular (integral) parts, we find $M_{j}$ and $a_{j}$ from the singular parts. From the regular parts, we have:

$$
\dot{\mu}_{r}(T)=\rho(X(T)) \dot{X}(T), \quad \dot{\nu_{r}}(T)=X(T) \rho(X(T)) \dot{X}(T) .
$$

From these relations, we find $X(T)$ and, finally, $\rho(x)$.

\section{Acknowledgments}

The research of S. Avdonin was supported in part by the National Science Foundation, grant DMS 1411564 and by the Ministry of Education and Science of Republic of Kazakhstan under the grant No. 4290/GF4.

\section{References}

[1] Gerasimenko N.I., Pavlov B.S. Scattering problems on noncompact graphs, Teoret. Mat. Fiz., 1988, 74, P. 345-359. Eng. transl., Theoret. and Math. Phys., 1988, 74, P. 230-240.

[2] Gerasimenko N.I. Inverse scattering problem on a noncompact graph. Teoret. Mat. Fiz., 1988, 75, P. 187-200. Translation in Theoret. and Math. Phys., 1988, 75, P. 460-470.

[3] Berkolaiko G., Kuchment P. Introduction to Quantum Graphs (Mathematical Surveys and Monographs vol 186). Providence, RI: American Mathematical Society, 2013

[4] Avdonin S. Control problems on quantum graphs. Analysis on Graphs and Its Applications, Proceedings of Symposia in Pure Mathematics, AMS, 2008, 77, P. 507-521.

[5] Avdonin S., Kurasov P. Inverse problems for quantum trees. Inverse Problems and Imaging, 2008, 2 (1), P. 1-21.

[6] Avdonin S., Bell J. Determining a distributed conductance parameter for a neuronal cable model defined on a tree graph. Journal of Inverse Problems and Imaging, 2015, 9 (3), P. 645-659.

[7] Avdonin S., Choque Rivero A., Leugering G., and Mikhaylov V. On the inverse problem of the two velocity tree-like graph. Zeit. Angew. Math. Mech., 2015, 1-11. DOI 10.1002 zamm.201400126.

[8] Avdonin S. and Edward J. Inverse problems for networks of vibrating strings with attached masses. SIAM Journal of Control and Optimization, submitted.

[9] Hansen S. and Zuazua E. Exact controllability and stabilization of a vibrating string with an interior point mass. SIAM J. Control Optim., 1995, 33 (5), P. 1357-1391.

[10] Avdonin S., Avdonina N. and Edward J. Boundary inverse problems for networks of vibrating strings with attached masses. Proceedings of Dynamic Systems and Applications, 2016, 7, P. 41-44. 